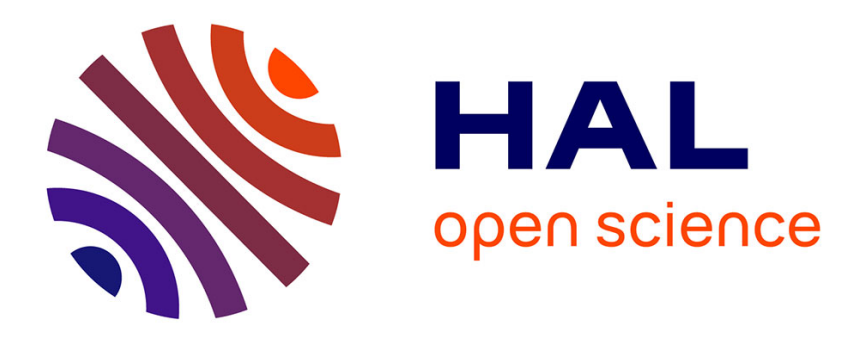

\title{
Le fonds Henri Cordier de l'université Keiō, Tōkyō Frédéric Girard
}

\section{To cite this version:}

Frédéric Girard. Le fonds Henri Cordier de l'université Keiō, Tōkyō. Bulletin de l'Ecole française d'Extrême-Orient, 2012, 99, pp.385-390. 10.3406/befeo.2012.6160 . halshs-01890009

\section{HAL Id: halshs-01890009 \\ https://shs.hal.science/halshs-01890009}

Submitted on 8 Oct 2018

HAL is a multi-disciplinary open access archive for the deposit and dissemination of scientific research documents, whether they are published or not. The documents may come from teaching and research institutions in France or abroad, or from public or private research centers.
L'archive ouverte pluridisciplinaire HAL, est destinée au dépôt et à la diffusion de documents scientifiques de niveau recherche, publiés ou non, émanant des établissements d'enseignement et de recherche français ou étrangers, des laboratoires publics ou privés. 


\section{Le fonds Henri Cordier de l'université Keiō, Tōkyō}

Frédéric Girard

Citer ce document / Cite this document :

Girard Frédéric. Le fonds Henri Cordier de l'université Keiō, Tōkyō. In: Bulletin de l'Ecole française d'Extrême-Orient. Tome 99, 2012. pp. 385-390;

doi : https://doi.org/10.3406/befeo.2012.6160

https://www.persee.fr/doc/befeo_0336-1519_2012_num_99_1_6160

Fichier pdf généré le 08/05/2018 


\title{
Le fonds Henri Cordier de l'université Keiō, Tōkyō
}

\author{
Frédéric GIRARD*
}

Le fonds Henri Cordier dont il est question ici fait partie d'une bibliothèque, le Eisei bunko 永青文庫, ayant appartenu à la grande famille de la haute noblesse, Hosokawa 細川家, dont les chefs étaient seigneurs du fief de Kumamoto 熊本藩. Il a été confié au fonds appelé Shidō bunko 斯道文庫, «Bibliothèque humaniste » de la bibliothèque Keiō. Ce dernier a été fondé à Fukuoka en 1938, en tant que personne juridique. La bibliothèque Shidō, qui a subi les bombardements de la Seconde Guerre mondiale en 1945, a été dissoute l'année suivante : elle comptait 70000 ouvrages, qui ont été entreposés à la bibliothèque de l'école annexe Asō appartenant à la société minière Azabu. En 1950, elle a été confiée à l'université de Kyūshū, sous le nom de bibliothèque Asō d'Azabu 麻生麻布文庫. Elle a ensuite été donnée à l'université Keiō en 1958, à l'occasion de son centenaire, et inaugurée en 1960 dans le but de « collectionner et conserver des matériaux concertant les textes classiques japonais et orientaux afin de procéder à des investigations et des recherches les concernant ». La bibliothèque poursuit ses travaux d'acquisition, de conservation et d'informatisation de ses matériaux, qui s'élèvent à 163000 ouvrages, dont 48500 proviennent de donations, 1300 sont des photographies, et 6200 sont des microfilms.

Aujourd'hui, la bibliothèque Eisei est quant à elle divisée en deux : une partie, restée dans le Kyūshū, est entreposée au musée d'art de la préfecture de Kumamoto (objets d'art) ainsi qu'à la bibliothèque de l'université de Kumamoto (documents d'archives) ; l'autre, qui se trouve à Tōkyō, est entreposée dans le musée de la bibliothèque Eisei, situé à Mejiro, d'où émane le présent fonds, objet d'une quasi-donation de la part de la famille Hosokawa. Celle-ci a confié aux bons soins de l'université Keiō le fonds appelé Cordier, qui a donc rejoint le Shidō bunko. Si la famille Hosokawa et le musée de la bibliothèque Eisei en sont toujours propriétaires d'un point de vue juridique, l'université Keiō en a une sorte de monopole de fait, même s'il est toujours nécessaire d'obtenir l'autorisation formelle du musée pour le consulter. Une petite partie du fonds Cordier est restée au musée Eisei bunko, pour les besoins des expositions.

Ce fonds Eisei bunko a été alimenté en 1967 par les 28000 volumes en provenance de l'ancien fonds de la bibliothèque Tandō 坦堂文庫, constitué d'ouvrages en chinois qu'a légués le professeur Kojō Sadakichi 古城貞吉, du Shidō bunko. Le fonds Henri Cordier a été l'objet d'une donation qui a immédiatement suivi en 1973. Il provient bien entendu du fonds personnel de l'auteur (1849-1925), orientaliste français réputé pour ses travaux en bibliographie orientale ainsi que, accessoirement, pour ses goûts raffinés dans plusieurs domaines des arts, des lettres (il a été président de la société des études stendhaliennes) et des divertissements, parmi lesquels l'alpinisme. On peut bien entendu se demander par quel concours de circonstances ce fonds est arrivé en terre japonaise, dans l'une de ses universités les plus réputées, l'université privée Keiō, fondée par Fukuzawa Yukichi 福沢諭吉 (1835-1901), illustre penseur, réformateur éclairé et audacieux

* Directeur d'études à l'École française d'Extrême-Orient. 
promoteur des sciences, de la libre pensée et d'un humanisme dont le Japon moderne est encore fortement marqué. Nul Japonais n'ignore son nom, puisque les actuels billets de banque de dix mille yens sont à son effigie. Le marquis Hosokawa Moritatsu 細川 護立侯 (1883-1970) a acquis le fonds lors d'un passage fortuit à Paris, au moment où l'épouse de Cordier, $\mathrm{M}^{\text {me }}$ Marguerite Baudry, a mis en vente une partie de la bibliothèque de son mari. Il a dû l'acquérir à prix d'or car il est vraisemblable que l'Institut de France, dont Henri Cordier était membre libre, devait convoiter cette immense bibliothèque de bibliophile. D'ailleurs, la bibliothèque de l'Institut de France possède également un fonds Henri Cordier. Celui-ci est constitué de 86 volumes, portefeuilles ou boîtes, d'ouvrages datant des XVII ${ }^{\mathrm{e}}$-XXe siècles (MS 5401-5486). Mais qui sait que l'université Keiō possède les perles les plus rares et précieuses de cette bibliothèque inestimable?

Un catalogue, complet et fort bien fait, a été dressé de cette bibliothèque. Les ouvrages de Cordier portent un signe distinctif sur la reliure extérieure, en l'espèce du caractère $\mathrm{Kō}$ 高 (ch. Gao), diminutif sino-japonais du nom de Cordier, et certains volumes ont la reliure gravée de l'insigne de la famille Hosokawa, sous la forme de barres horizontales blanches sur fond noir. Les conservateurs de la Bibliothèque humaniste se sont attelés avec zèle et compétence au travail de catalogage de ce fonds, une des spécialités pour lesquelles les Japonais sont réputés dans le monde entier. Le travail a été accompli en quelque cinq années et a donné lieu à une publication de 147 pages.

Henri Cordier naît en 1849, à la Nouvelle Orléans, dans une famille française d'hommes d'affaires, et il revient à Paris trois années après. Il y poursuit ses études et, à l'âge de quinze ans, effectue un séjour en Angleterre afin de s'y perfectionner dans la langue. À son retour, en 1869 , il renonce à entrer à l'École nationale des chartes pour se rendre en Chine, comme employé de la compagnie Russel établie à Shanghai, suivant les conseils de son père qui a travaillé dans une banque en Chine. Durant les sept années que dure son séjour, il se met à l'étude du chinois. Il devient bibliothécaire honoraire de la section du nord de la Chine de la Royal Asiatic Society en 1871 ; il s'attèle à son catalogage, se livrant dans le même temps à sa dévorante manie de collectionner des ouvrages d'orientalisme : l'infortune veut que la collection qu'il avait réunie périsse dans le vaisseau qui l'achemine vers l'Europe en 1877 ! Il garde de ce premier long séjour des amitiés durables dans tous les milieux, qui lui seront précieuses : Samuel Wells Williams (1812-1884), un missionnaire américain arrivé au Japon en même temps que le commodore Matthew Perry (1794-1858), l'archimandrite Palladius (1817-1878), Petre Ivanovitch Koporoff de son nom de laïc, ou le biologiste français Armand David (1826-1900). À son retour en France, en 1876, il devient secrétaire de la délégation éducative du gouvernement chinois pour les étudiants chinois en Europe et, à ce titre, ajourne son voyage en Chine. En 1881, sa valeur scientifique et universitaire est reconnue par le président de l'École nationale des langues orientales vivantes, Charles Schefer (1820-1898), et il succède à Jean-Pierre Guillaume Pauthier (1801-1873) avec une chaire d' ' histoire, géographie et législation des États d'Extrême-Orient ». Il y gagne auprès des étudiants la réputation d'une encyclopédie vivante que son immense barbe rend encore plus sympathique. Il sait également se montrer entreprenant dans l'organisation des congrès, des institutions scientifiques et des recherches. En 1882, il inaugure la Revue de l'Extrême-Orient qui couvre tous les domaines de l'Asie orientale mais qui ne vivra que cinq années et ne tirera que trois numéros. En 1890, il fonde avec le savant hollandais Gustave Schlegel la revue T'oung Pao 通報, publiée à Leiden, qui deviendra rapidement très prestigieuse, et à laquelle il s'attèlera sa vie durant. 
Orientaliste chevronné, Cordier donne des contributions scientifiques notables dans les domaines de l'histoire et de la géographie. Il est naturellement couvert d'honneurs en raison de la diversité de ses compétences : membre honoraire de la Royal Asiatic Society en 1893, membre de l'Académie des inscriptions et belles-lettres en 1908, membre honoraire de la Société géographique de Genève en 1909, membre de la Société de géographie de Rome en 1916, vice-président de la Société asiatique, président de la Société d'ethnologie, en 1918, membre correspondant de l'Académie d'Angleterre, en 1921, président de la Société de géographie en 1924. Cordier est aussi président de la société Stendhal et ami du peintre Gustave Caillebotte (1848-1894) qui a laissé de lui un portrait à l'huile, conservé au musée d'Orsay (1883).

Henri Cordier est l'auteur bien connu d'une impressionnante Bibliographie chinoise (Bibliotheca Sinica), qu'il a confectionnée en 1881-1885 et rééditée en la complétant très largement en 1902-1908, avant de lui adjoindre d'importants suppléments en $1924^{1}$. Il a de plus compilé une très importante Bibliographie japonaise (Bibliotheca Japonica) parue en $1912^{2}$. Il a rédigé une Bibliotheca Indosinica en 1912-1915. Il est également l'auteur de toute une série d'ouvrages bibliographiques portant sur plusieurs domaines des études orientales, qui font le régal des lecteurs, orientalistes ou non, par leur utilité et le plaisir qu'on a à les consulter, tant son auteur a su les agrémenter avec un bonheur inégalé d'une documentation aussi variée que pittoresque et scientifique. Mentionnons, par exemple, son Histoire générale de la Chine et de ses relations avec les pays étrangers depuis les temps les plus anciens jusqu'à la chute de la dynastie manchoue ${ }^{3}$, laquelle collectionne les citations sur la Chine que Cordier a puisées parmi les récits de voyages, les lettres, les histoires, les encyclopédies, les recueils de pensées, les ouvrages philosophiques, etc. Mais c'est à l'ensemble des relations et des rapports entre les cultures de l'Est et de l'Ouest qu'il s'intéresse dans des ouvrages qui n'ont nullement vieilli et qui gardent toute leur valeur documentaire et scientifique : il traduit The Book of Ser Marco Polo, en 1903, Cathay and the Way Thither, en 1913-1916, Les Voyages en Asie au $X I V^{*}$ siècle du bienheureux Frère Odoric de Pordenone Religieux de Saint-François, en 1891, Les Merveilles de l'Asie par le Père Jourdain Catalani de Sévérac de l'ordre des Frères Prêcheurs, évêque de Columbum (XIV' siècle), en 1925. Il compile un Recueil de voyages et de documents pour servir à l' histoire de la géographie depuis le XIIr' jusqu' à la fin du XVI' siècle, entre 1882 et 1923. Il s'intéresse également à l'histoire diplomatique de la Chine et rédige L'Expédition de Chine de 1857-1858. Histoire diplomatique, en 1905, et enfin une Histoire générale de la Chine et de ses relations avec les pays étrangers, en 1920-1921, que nous avons évoquée. Collectionneur invétéré et impénitent d'ouvrages rares et précieux, au cours d'une vie de trois quarts de siècle particulièrement bien remplie, il a su monter une bibliothèque du plus grand prix pour les études orientales, et surtout chinoises, son domaine de prédilection. C'est un total de cinq mille volumes réunis avec patience, labeur et discernement que le marquis Hosokawa a décidé de confier à la Bibliothèque humaniste de l'université Keiō, pour des raisons de conservation

1. Bibliotheca Sinica. Dictionnaire bibliographique des ouvrages relatifs à l'Empire chinois, $2^{\text {de }}$ édition, revue, corrigée et considérablement augmentée, volume 1, Paris, Librairie orientale et américaine, E. Guimoto, 1904 ; Bibliotheca Sinica. Dictionnaire bibliographique des ouvrages relatifs à l'Empire

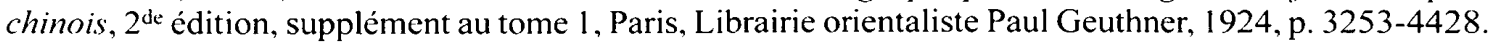

2. Bibliotheca Japonica. Dictionnaire bibliographique des ouvrages relatifs à l'Empirc japonais rangés par ordre chronologique jusqu'à 1870 suivi d'un appendice renfermant la liste alphabétique des principaux ouvrages parus de 1870 à 1912, Paris, Ernest Leroux, 1912.

3. Paris, Librairie Paul Geuthner, 1920. 
tout d'abord, mais aussi et surtout pour le bien et le profit des étudiants, chercheurs et professeurs se vouant aux études classiques de l'orientalisme, du Moyen-Orient jusqu'à l'Extrême-Orient, en ouvrant volontairement ce fonds à un public universitaire de la meilleure qualité. Il comprend des incunables remontant jusqu'au $\mathrm{XV}^{\mathrm{e}}$ siècle, des ouvrages d'orientalisme en langues occidentales dans les domaines littéraire, historique, juridique, lexicologique, archéologique, d'histoire de l'art, des relations de voyages intéressant l'Afrique, l'Amérique, le Japon, la Chine. Nombre de ces ouvrages portent la signature de ses éminents collègues et amis, Paul Pelliot, Édouard Chavannes, Gustave Schlegel, Emil Bretsteiner, Aurel Stein, William Woodville Rockhill, Charles Joseph de Harlez, Camille Imbault-Huart, Berthold Laufer.

Le marquis Hosokawa Moritatsu fait partie, comme on l'a dit, de cette haute noblesse du sud du Japon, de l'île de Kyūshū, qui a pour ancêtre Ashikaga Yoshisue 足利義季 de la lignée des Seiwa Genji 清和源氏, personnage qui a pris pour nom Hosokawa Jirō 細川二郎 à partir du toponyme où il s'était établi dans la province de Mikawa. Elle s'est illustrée auprès des shōgun Ashikaga Takauji 足利尊氏 (1305-1358) et Yoshimitsu 足利義満 (1358-1408) et, dans l'histoire, par les plus grands noms : Hosokawa Fujitaka 細川藤孝 ou Yūsai Genshi 幽斎玄旨 (1534-1610) a été le fin politicien et l'éminent homme de lettres et d'art du début de l'époque Tokugawa que l'on sait, fils du shōgun Yoshiharu 足利義晴 (1511-1550), promu daimyō de Tango par Oda Nobunaga 織田信長 (1534-1582) ; devenu religieux après l'assassinat de ce dernier, il lègue son fief à son fils et s'adonne à des activités littéraires, en composant des écrits sur les arts martiaux, le Nō, les rituels de cour et la poésie, tout en laissant des récits des campagnes militaires de Toyotomi Hideyoshi 豊臣秀吉 (1537-1598). Son fils Hosokawa Tadaoki 細川忠興 (1563-1645), devenu ami du shōgun Tokugawa Hidetada 徳川秀忠 (1579-1632), est lui aussi homme de lettres versé en poésie, en Nō ainsi que dans les traditions martiales et du thé - il est l'un des derniers disciples de Sen no Rikyū, et auteur de plusieurs traités sur le thé sous le nom de Hosokawa Sansai 細川三齋. Il s'est illustré par des édifications architecturales et artistiques du plus grand raffinement et a été gratifié de sa fidélité par Tokugawa Iyeyasu 徳川家康 (1542-1616) d'une partie des fiefs de Buzen et de Bungo. Son épouse, Gracia 細川ガラシャ(1563-1600), Akechi Tama 明智玉, est cette chrétienne convertie à l'instigation des pères jésuites les plus en vue, en 1587, auteur d'écrits et de traductions remarquables, et dont la vie est l'incarnation d'une dévotion sans faille. Hosokawa Tadatoshi 細川忠利 (1586-1641), un fils de Tadaoki et lui aussi versé dans les arts martiaux, invite un guerrier de l'envergure de Miyamoto Musashi 台本武蔵(1584 ?-1645), et s'illustre dans la répression de Shimabara (1631-1632). Il écrit des pièces de nō et est le premier au Japon à avoir fait état de la fabrication de vin au Japon. Hosokawa Yukitaka 細川行孝 (1637-1690) a le premier décrit dans l'archipel les systèmes hydrauliques de canalisation en surface et le mécanisme d'extraction des sources. Hosokawa Shigekata 細川重賢 (1720-1785), seigneur éclairé des fiefs de Kumamoto et de Wakayama, féru de science, de médecine et de technique, de botanique, de généalogie et d'encyclopédisme, n'a cessé d'alimenter les collections des Hosokawa, engrangées maintenant en bonne partie au musée Eisei bunko, sur le site de la résidence des Hosokawa à Mejirodai, qui a été établie en 1892 (résidence européenne) et 1893 (résidence japonaise). Les carrières des Hosokawa successifs donnent une idée des biens qui s'y sont accumulés, parmi lesquels de nombreux trésors nationaux et biens culturels importants, destinés à être exposés pour le public : des écrits littéraires, lc Dit de Ise, des vêtements d'actcurs de nō, des écrits historiques sur la province de Higo, des cartes, des objets rares japonais, chinois ou venus d'Occident. 
Le grand-père de Moritatsu a été un Premier ministre japonais de renom. Moritatsu est connu comme historien de l'art, comme collectionneur d'œuvres artistiques aussi bien anciennes que modernes, et pour avoir mené et promu une politique culturelle et scientifique d'envergure. Il est né à Tōkyōo ; c'est le quatrième fils de Hosokawa Morihisa 細川護久 (1839-1893), seigneur du fief de Kumamoto ; il commence jeune à collectionner des œuvres d'art, de Hakuin en particulier, occupe des positions importantes dans le nouveau gouvernement de Meiji et perpétue une politique progressiste dans son fief, autant sur les plans économique et politique que culturel et humaniste. Il poursuit des études de droit à l'université impériale de Tōkyō (1906), sans aller jusqu'au bout du cursus. Il participe à la revue Shirakaba (1910). En 1914, il devient marquis et occupe des fonctions de distinction : membre de la chambre des Pairs (Kizokuin giin), président de la Société de géographie de Tōkyō et président de la Société pour la conservation des trésors nationaux. On le voit participer à la cérémonie d'intronisation de l'empereur Taishō (1915), édifier un pavillon de nō à Kōjimachi (1916-1919), acquérir des peintures de Cézanne ou de Renoir, notamment à Paris (1920-1922), devenir président de l'Association d'homélie de Shōtoku taishi (1925). Il séjourne un certain temps à Paris (1925-1926), où il continue à monter ses collections de peintures et acquiert la bibliothèque de Cordier. Il s'attache à restaurer le musée de la famille impériale et devient président de la Société pour la conservation des trésors nationaux (1929). Il publie les œuvres complètes du moine Zen Takuan. En 1943, il transporte les œuvres d'art qu'il possède dans ses résidences à Kumamoto et à Karuizawa. Au lendemain de la Seconde Guerre mondiale, il est conseiller pour les trésors du Shōsōinen 1946, du musée national de Tōkyō en 1947, et trois années plus tard, membre de la conservation des biens culturels. Il fonde la bibliothèque Eisei en 1950, devient président du musée national de Tōkyō en 1952, puis administrateur en chef de la Bibliothèque orientale (Tôyō bunko) et chef de la délégation de coopération pour la protection des vestiges en Nubie. Moritatsu était particulièrement versé dans les sciences et les techniques ainsi que dans la géographie et l'alpinisme. On peut conjecturer que ce sont ces derniers aspects qui ont attiré son attention sur la bibliothèque de Cordier (lui-même féru dans ces domaines), laquelle comprend, en effet, un nombre important d'ouvrages sur la géographie, l'alpinisme et l'histoire des sciences et des techniques.

La bibliothèque Cordier comprend des ouvrages rédigés dans les principales langues occidentales se rapportant à des travaux scientifiques, ethnologiques et bibliographiques. Ils couvrent des domaines généraux (Manuel du libraire et de l'amateur de livres de Jacques-Charles Brunet, 1922), aussi bien que des études particulières (The Chinese missionary gleaner, 1850-1856), ou d'intérêt historique ou social (Socialism, a summary and interpretation of socialists principles, de John Spargo, 1919), de nature méthodologique (Manuel de numismatique orientale de l'Antiquité et du Moyen-Âge de J. de Morgan, 1923-1924). Des collections comme les Annales du Musée Guimet sont entreposées au Shidō bunko, mais le lecteur sera contraint de se rendre au Eisei bunko afin d'y trouver et consulter les tout premiers numéros ; en revanche, la série des Congrès International des Orientalistes se trouve en entier au Shidō bunko (années 1873 et suivantes).

La curiosité scientifique de Henri Cordier suivait tout d'abord sa logique propre, qu'il est intéressant de saisir dans sa singularité comme dans ses diverses facettes; le bibliothécaire peut être désarçonné et ne pas toujours s'y retrouver. Le catalogue actuellement dressé par le Shidō bunko, qui est notre meilleur guide, classifie les livres et documents de Cordier de la manière suivante, en se conformant aux sigles les plus courants au Japon : 0. Ouvrages de référence généraux ; I. Europe ; II. Asie, Asie orientale, Pacifique ; 
III. Chine ; IV. Japon ; V. Iran, Turquie ; VI. Inde ; VII. Amérique ; VIII. Mandchourie et Mongolie ; IX. Afrique ; X. Australie ; YW. Livres de grandes dimensions ; A. Livres anciens européens ; C. Congrès ; D. Dictionnaires ; L. Droit ; M. Alpinisme ; S. Sport ; P. Périodiques.

Chaque section est à nouveau divisée en sous-sections, au nombre d'une dizaine ou d'une quinzaine, ce qui permet de se repérer assez facilement dans cet ensemble. Le catalogue est clos par un pratique index des auteurs.

Les ouvrages de référence généraux sont par exemple regroupés sous dix-huit chefs : 1. Ouvrages généraux (Bibliographies \& catalogues, Ouvrages collectifs, Mélanges), 2. Géographie, 3. Anthropologie, 4. Astronomie, 5. Histoire naturelle, 6. Archéologie, 7. Histoire, 8. Biographie, 9. Philosophie, 10. Religion, 11. Art (Ouvrages généraux, Peinture, Architecture \& sculpture, Poterie \& porcelaine, Textiles, Catalogues \& collections, Arts décoratifs, Antiquité), 12. Philologie, 13. Littérature, 14. Économie, 15. Communication, 16. Problèmes sociaux, 17. Armée (lire : 17 et non pas 18).

C'est à explorer cette bibliothèque, parvenue au Japon grâce à des concours de circonstances étonnants, que nous invite son catalogue. Le lecteur y découvrira toute la richesse du savoir d'un érudit français qui a joué son rôle à la charnière des $X_{I} X^{e}$ et $\mathrm{xx}^{\mathrm{e}}$ siècles et continue de nourrir les bibliographies concernant l'Extrême-Orient, tant son exploration dans ces domaines est allée loin et reste souvent inégalée. 